\title{
Inverse Estimates for Nonhomogeneous Backward Heat Problems
}

\author{
Tao Min, ${ }^{1}$ Weimin Fu, ${ }^{1}$ and Qiang Huang ${ }^{2}$ \\ ${ }^{1}$ School of Science, Xian University of Technology, Xian, Shaanxi 710054, China \\ ${ }^{2}$ State Key Laboratory of Eco-Hydraulic Engineering in Shaanxi, Xian University of Technology, Xian, Shaanxi 710048, China
}

Correspondence should be addressed to Tao Min; mintao@xaut.edu.cn

Received 16 September 2013; Accepted 12 January 2014; Published 2 March 2014

Academic Editor: Zhong Bo Fang

Copyright (C) 2014 Tao Min et al. This is an open access article distributed under the Creative Commons Attribution License, which permits unrestricted use, distribution, and reproduction in any medium, provided the original work is properly cited.

We investigate the inverse problem in the nonhomogeneous heat equation involving the recovery of the initial temperature from measurements of the final temperature. This problem is known as the backward heat problem and is severely ill-posed. We show that this problem can be converted into the first Fredholm integral equation, and an algorithm of inversion is given using Tikhonov's regularization method. The genetic algorithm for obtaining the regularization parameter is presented. We also present numerical computations that verify the accuracy of our approximation.

\section{Introduction}

Inverse heat conduction problems (IHCPs) appear in many important scientific and technological fields [1-6]. Hence analysis, design implementation, and testing of inverse algorithms are also great scientific and technological interest. In this paper we will examine some inversion techniques in order to estimate the initial temperature distribution of an inverse nonhomogeneous heat conduction problem; this problem is called the backward heat problem, the backward Cauchy problem, or the final value problem. As is known, the nonhomogeneous problem is severely ill-posed; that is, solutions do not always exist, and in the case of existence, these do not depend continuously on the given data. In fact, from small noise contaminated physical measurements, the corresponding solutions have large errors. It makes it difficult to do numerical calculations. Hence, a regularization is in order. Lattes and Lions, in [7], regularized the problem by adding a "corrector" to the main equation. Gajewski and Zacharias [8] considered a similar problem. Nakamura et al. [9] used transformation techniques to solve the initial inverse problem in heat conduction and Al-Khalidy [10] dealt with the problem numerically. For a comprehensive review of the literature and summary of various approaches in the field of inverse heat conduction problems, one can consult the books by Beck et al. [11] and by Hensel [12]. Although there are many papers on the linear homogeneous case of the backward problem, we only find a few papers on the nonhomogeneous case, such as in $[13,14]$.

The plan of this paper is as follows. In Section 2, we formulate a one-dimensional IHCP. In Section 3, we show that this problem can be converted into the first Fredholm integral equation, the numerical algorithm is derived, and an algorithm of inversion is also given using Tikhonov's regularization method. We use the genetic algorithm for the choice of the regularization parameter in Section 4. Section 5 presents numerical experiments to show the efficiency of the proposed method. Section 6 ends this paper with a brief discussion on some numerical aspects.

\section{Mathematical Model}

2.1. The Direct Problem. The direct (forward) problem consists of a transient heat conduction problem in a slab with adiabatic boundary condition and initially at a temperature denoted by $f(x)$. 
The mathematical formulation of this problem is given by the following nonhomogeneous heat equation:

$$
\begin{array}{cl}
\frac{\partial u}{\partial t}-k \frac{\partial^{2} u}{\partial x^{2}}=\varphi(x, t), & 0<x<L, t>0, \\
u(0, t)=0, & t>0, \\
u(L, t)=0, & t>0, \\
u(x, 0)=f(x), & 0 \leq x \leq L,
\end{array}
$$

where $u(x, t)$ (temperature), $\varphi(x, t)$ (source term), $f(x)$ (initial condition), $x$ (spatial variable), and $t$ (time variable) are dimensionless quantities and $k$ denotes the dispersion coefficient.

For the direct problem where the initial condition $f(x)$ is specified, the problem given by (1) and (2) is concerned with the determination of the temperature distribution $u(x, t)$ in the interior region of the solid as a function of time and position.

2.2. Inverse Problem. For the inverse problem, the initial condition $f(x)$ is regarded as being unknown. In addition, an overspecified condition is also considered available. To estimate the unknown coefficient $f(x)$, the additional information,

$$
u(x, T)=g(x)
$$

is given at time $T$, over a specified space interval $0 \leq$ $x \leq L$. We note that the measured overspecified condition $u(x, T)=g(x)$ should contain measurement errors. Therefore the inverse problem can be stated as follows: estimate the unknown function $f(x)$ by utilizing the above-mentioned measured data.

\section{Algorithm Analysis}

The solution of the direct problem for a given initial condition $f(x)$ is explicitly obtained using separation of variables for $0<x<L, t \geq 0$. Consider the initial boundary problems (1) and (2); we look for solutions $u$ in the form $u(x, t)=$ $T(t) X(x)$. We look at the following eigenvalue problem:

$$
\begin{gathered}
X^{\prime \prime}+\lambda X=0 \\
X(0)=X(L)=0 .
\end{gathered}
$$

The eigenvalues and the corresponding eigenfunctions are

$$
\lambda=\left(\frac{n \pi}{L}\right)^{2}, \quad X_{n}(x)=\sin \frac{n \pi}{L} x
$$

Now we set

$$
u(x, t)=\sum_{n=1}^{\infty} T_{n}(t) \sin \frac{n \pi}{L} x .
$$

Formally computing $u_{t}$ and $u_{x x}$ and substituting into (1), we get

$$
\begin{aligned}
\varphi(x, t) & =u_{t}-k u_{x x} \\
& =\sum_{n=1}^{\infty}\left[T_{n}^{\prime}(t)+k\left(\frac{n \pi}{L}\right)^{2} T_{n}(t)\right] \sin \left(\frac{n \pi x}{L}\right) .
\end{aligned}
$$

Hence expanding $f$ and $\phi$ into the following Fourier series:

$$
\begin{gathered}
\varphi(x, t)=\sum_{n=1}^{\infty} \varphi_{n}(t) \sin \left(\frac{n \pi x}{L}\right), \\
f(x)=\sum_{n=1}^{\infty} f_{n} \sin \left(\frac{n \pi x}{L}\right),
\end{gathered}
$$

where

$$
\begin{gathered}
\varphi_{n}(t)=\frac{2}{L} \int_{0}^{L} \varphi(x, t) \sin \left(\frac{n \pi x}{L}\right) d x, \\
f_{n}=\frac{2}{L} \int_{0}^{L} f(x) \sin \left(\frac{n \pi x}{L}\right) d x,
\end{gathered}
$$

we get

$$
\begin{gathered}
\sum_{n=1}^{\infty}\left[T_{n}^{\prime}(t)+k\left(\frac{n \pi}{L}\right)^{2} T_{n}(t)\right] \sin \left(\frac{n \pi x}{L}\right) \\
=\sum_{n=1}^{\infty} \varphi_{n}(t) \sin \left(\frac{n \pi x}{L}\right) .
\end{gathered}
$$

The uniqueness of the Fourier expansion leads to the family of $O D E$ 's:

$$
T_{n}^{\prime}(t)+k\left(\frac{n \pi}{L}\right)^{2} T_{n}(t)=\varphi_{n}(t) .
$$

In addition,

$$
u(x, 0)=\sum_{n=1}^{\infty} T_{n}(0) \sin \frac{n \pi}{L} x=\sum_{n=1}^{\infty} f_{n} \sin \left(\frac{n \pi x}{L}\right)=f(x),
$$

so that

$$
T_{n}(0)=f_{n}, \quad n \geq 1 .
$$

Solving (11)-(13), we find

$$
\begin{aligned}
T_{n}(t)= & f_{n} e^{-k(n \pi / L)^{2} t}+\int_{0}^{t} e^{-k(n \pi / L)^{2}(t-s)} \varphi_{n}(s) d s, \\
u(x, t)= & \sum_{n=1}^{\infty} f_{n} e^{-k(n \pi / L)^{2} t} \sin \left(\frac{n \pi x}{L}\right) \\
& +\sum_{n=1}^{\infty} \sin \left(\frac{n \pi x}{L}\right) \int_{0}^{t} e^{-k(n \pi / L)^{2}(t-s)} \varphi_{n}(s) d s, \\
u(x, t) & -\sum_{n=1}^{\infty} \sin \left(\frac{n \pi x}{L}\right) \int_{0}^{t} e^{-k(n \pi / L)^{2}(t-s)} \varphi_{n}(s) d s \\
= & \int_{0}^{L} K(x, y, t) f(y) d y,
\end{aligned}
$$


where $K(x, y, t)=(2 / L) \sum_{n=1}^{\infty} e^{-(n \pi)^{2} k t / L^{2}} \sin (n \pi x / L) \sin (n \pi y /$ $L) . K(x, y, t)$ is an infinite series; numerically we cannot handle infinite sums. Limit the sum to a finite number of expansion terms 200 which guarantees the convergence of the series. So

$$
K(x, y, t) \approx \frac{2}{L} \sum_{n=1}^{200} e^{-(n \pi)^{2} k t / L^{2}} \sin \left(\frac{n \pi x}{L}\right) \sin \left(\frac{n \pi y}{L}\right) .
$$

Thus initial inverse problem is reduced to solving integral equation of the first kind:

$$
w(x)=\int_{0}^{L} K(x, y, T) f(y) d y
$$

where

$$
\begin{aligned}
w(x) & =u(x, T)-\sum_{n=1}^{\infty} a_{n} \sin \left(\frac{n \pi x}{L}\right) \\
& =g(x)-\sum_{n=1}^{\infty} a_{n} \sin \left(\frac{n \pi x}{L}\right) \\
a_{n} & =\frac{2}{L} \int_{0}^{T} e^{-k(n \pi / L)^{2}(T-s)}\left[\int_{0}^{L} \varphi(x, s) \sin \left(\frac{n \pi x}{L}\right) d x\right] d s \\
& =\int_{0}^{L} \int_{0}^{T} \frac{2}{L} e^{-k(n \pi / L)^{2}(T-s)} \phi(x, s) \sin \left(\frac{n \pi x}{L}\right) d s d x \\
& =\int_{0}^{L} \int_{0}^{T} g(x, s, n) d s d x,
\end{aligned}
$$

where $g(x, s, n)=(2 / L) e^{-k(n \pi / L)^{2}(T-s)} \phi(x, s) \sin (n \pi x / L)$. Since $a_{n}$ is a double integral, it is difficult to determine the exact value. The authors try to seek its approximation by complex trapezoid formula.

Given that the interval $[0, L]$ is equally subdivided into $m$ subintervals, $\left.\left\{\left[x_{i-1}, x_{i}\right]\right\}\right|_{i=1} ^{m}$, with equally spaced sampling points $x_{i}=i h$ where $i=0,1,2, \ldots, m$. Also, assume that the interval $[0, T]$ is equally subdivided into $l$ subintervals, $\left.\left\{\left[s_{j-1}, s_{j}\right]\right\}\right|_{j=1} ^{l}$, with equally spaced sampling points $s_{j}=j k$ where $j=0,1,2, \ldots, l$. The composite Trapezoidal rule is

$$
\begin{gathered}
a_{n} \approx \frac{1}{4} h k(g(0,0, n)+g(L, 0, n)+g(0, T, n)+g(L, T, n) \\
+2 \sum_{i=1}^{m-1} g\left(x_{i}, 0, n\right)+2 \sum_{i=1}^{m-1} g\left(x_{i}, T, n\right)
\end{gathered}
$$

$$
\begin{aligned}
& +2 \sum_{j=1}^{l-1} g\left(0, s_{j}, n\right)+2 \sum_{j=1}^{l-1} g\left(L, s_{j}, n\right) \\
& \left.+4 \sum_{j=1}^{l-1}\left(\sum_{i=1}^{m-1} g\left(x_{i}, s_{j}, n\right)\right)\right) .
\end{aligned}
$$

Approximation of $a_{n}$ can be obtained by the above formula.

The first step in the numerical treatment used in this research consists in discretization of (16) by the quadrature formula. The interval $[0, L]$ can be subdivided into $N$ equal intervals of width $h=\Delta y=L / N$. Let $y_{0}=0$ and $y_{j}=j \Delta y$, and since the variable is either $y$ or $x$, let $x_{0}=y_{0}=0$, $x_{N}=y_{N}=L$, and $x_{i}=i \Delta y$ (i.e., $\left.x_{i}=y_{i}\right)$. Also denote $f\left(x_{i}\right)$ as $f_{i}, w\left(x_{i}\right)$ as $w_{i}$, and $K\left(x_{i}, y_{j}, T\right)$ as $K_{i j}$. Now if the trapezoid rule is used to approximate the given equation, then

$$
\begin{aligned}
& w(x)=\int_{0}^{L} K(x, y, T) f(y) d y \\
& \approx \Delta y\left[\frac{1}{2} K\left(x, y_{0}, T\right) f\left(y_{0}\right)+K\left(x, y_{1}, T\right) f\left(y_{1}\right)\right. \\
& \\
&+\cdots+K\left(x, y_{N-1}, T\right) f\left(y_{N-1}\right) \\
&\left.+\frac{1}{2} K\left(x, y_{N}, T\right) f\left(y_{N}\right)\right],
\end{aligned}
$$

or, more tersely,

$$
\begin{aligned}
w(x) \approx \Delta y[ & \frac{1}{2} K\left(x, y_{0}, T\right) f_{0}+K\left(x, y_{1}, T\right) f_{1} \\
& \left.+\cdots K\left(x, y_{N-1}, T\right) f_{N-1}+\frac{1}{2} K\left(x, y_{N}, T\right) f_{N}\right] .
\end{aligned}
$$

There are $N+1$ values of $f_{i}$, as $i=0,1,2, \ldots, N$. Therefore the equation becomes a set of $N+1$ equations in $f_{i}$ :

$$
\begin{array}{r}
w_{i}=\Delta y\left[\frac{1}{2} K_{i 0} f_{0}+K_{i 1} f_{1}+\cdots K_{i(N-1)} f_{N-1}+\frac{1}{2} K_{i N} f_{N}\right] \\
i=0,1,2, \ldots, N
\end{array}
$$

which give the approximate solution to $f\left(x_{i}\right)$ at $x=x_{i}$. This may also be written in matrix form:

$$
K F=W
$$

where $K$ is the matrix of coefficients, 


$$
K=\Delta y \cdot\left[\begin{array}{cccc}
\frac{1}{2} K\left(x_{0}, y_{0}, T\right) & K\left(x_{0}, y_{1}, T\right) & \cdots & \frac{1}{2} K\left(x_{0}, y_{N}, T\right) \\
\frac{1}{2} K\left(x_{1}, y_{0}, T\right) & K\left(x_{1}, y_{1}, T\right) & \cdots & \frac{1}{2} K\left(x_{1}, y_{N}, T\right) \\
\ldots & \cdots & \cdots & \cdots \\
\frac{1}{2} K\left(x_{N}, y_{0}, T\right) & K\left(x_{N}, y_{1}, T\right) & \cdots & \frac{1}{2} K\left(x_{N}, y_{N}, T\right)
\end{array}\right],
$$

$F$ is the matrix of solutions,

$$
F=\left[f\left(y_{0}\right), \ldots, f\left(y_{i}\right), \ldots, f\left(y_{N}\right)\right]^{T},
$$

and $W$ is the matrix of the nonhomogeneous part:

$$
\begin{aligned}
W= & {\left[w\left(x_{0}\right), \ldots, w\left(x_{i}\right), \ldots, w\left(x_{N}\right)\right]^{T}, } \\
w\left(x_{i}\right)= & u\left(x_{i}, T\right)-\sum_{n=1}^{\infty} a_{n} \sin \left(\frac{n \pi x_{i}}{L}\right)=g\left(x_{i}\right) \\
& -\sum_{n=1}^{\infty} a_{n} \sin \left(\frac{n \pi x_{i}}{L}\right), \quad i=0,1,2, \ldots, N .
\end{aligned}
$$

The problem (22) is ill-posed in the sense that the inverse operator $K^{-1}$ of $K$ exists but is not continuous. Hence, although the problem (22) has a unique solution, solving it directly will not give a right solution. Indeed, the linear operator $K$ is so badly conditioned that any numerical attempt to directly solve (22) may fail.

In this note we describe the Tikhonov regularization for finding a stable approximate solution to a linear ill-posed problem represented in the form of an operator equation:

$$
K F=W,
$$

where, instead of the exact data $W$, noisy data $W_{\delta}$ is available with

$$
\left\|W-W_{\delta}\right\| \leq \delta
$$

In order to find a solution in stable manner, Tikhonov proposed to solve the following:

$$
F_{\alpha, \delta}=\underset{F \in R^{N+1}}{\arg \min } J_{\alpha}(F)=\left(\left\|K F-W_{\delta}\right\|_{2}^{2}+\alpha\|F\|_{2}^{2}\right),
$$

where $\alpha$ is the regularization parameter. The computation of the approximate solution $F_{\alpha, \delta}$ consists in solving the Euler equation corresponding to the functional $J_{\alpha}$. This equation has the following form:

$$
\left(K^{*} K+\alpha I\right) F_{\alpha, \delta}=K^{*} W_{\delta},
$$

where $K^{*}$ is the adjoint operator of $K$ and $I$ is the identity operator. The regularization solution $F_{\alpha, \delta}$ obviously depends on the parameter $\alpha$, the value of which directly affects the degree of approximation and the stability of the solution. From the viewpoint of the approximation degree, the smaller the better for the value of $\alpha$; however, from the viewpoint of the solution stability, the larger the better. So the key point for solving the problem is getting the value of $\alpha$.

In order to obtain an effectively approximate solution to the original ill-posed problem, it is particularly important to choose suitable parameter. If we do not have the information of $W$, we have to use an "a posteriori" parameter choice, where one uses results of the actual computation like the residual for computing a regularization parameter. The most widely used method is Morozov's discrepancy principle, where one takes $\alpha$ such that

$$
\left\|K F_{\alpha, \delta}-W_{\delta}\right\|_{2}=\delta .
$$

On how to solve the equation $\left\|K F_{\alpha, \delta}-W_{\delta}\right\|_{2}=\delta$, some researchers have developed some theories and methods to determine $\alpha[13,15-19]$. In the present paper, the genetic algorithm for obtaining the regularization parameter is presented.

\section{The Genetic Algorithm}

Genetic algorithms belong to the general category of stochastic global optimization methods [20-22]. They have their philosophical basis in a process found in nature related to the evolution of the different biological species. Based on Darwin's theory of evolution where the fittest individuals survive, while the less fit are eliminated, a simple genetic algorithm is basically composed of three operators: (i) selection, (ii) crossover, and (iii) mutation. The computational implementation of a genetic algorithm starts with random generation of a population of individuals where each individual is actually a chain composed of representations of binary numbers, sequentially, random values for design variables. Each individual in the population corresponds to a value of the objective function (fitness function),

$$
\lambda(\alpha)=\left|\left\|K F_{\alpha, \delta}-W_{\delta}\right\|_{2}-\delta\right|
$$

which is associated with a function of adaptability that is minimized.

With the fitness function values calculated for each individual in the population, the probability that this individual will be selected for crossover is determined. This probability is given by

$$
p_{i}=\frac{\lambda_{i}}{\sum_{i=1}^{n} \lambda_{i}}, \quad i=1,2, \ldots, n,
$$

where $\lambda_{i}$ is the fitness function, defined in (31), and $n$ is the population size. The fittest individuals have higher probability 


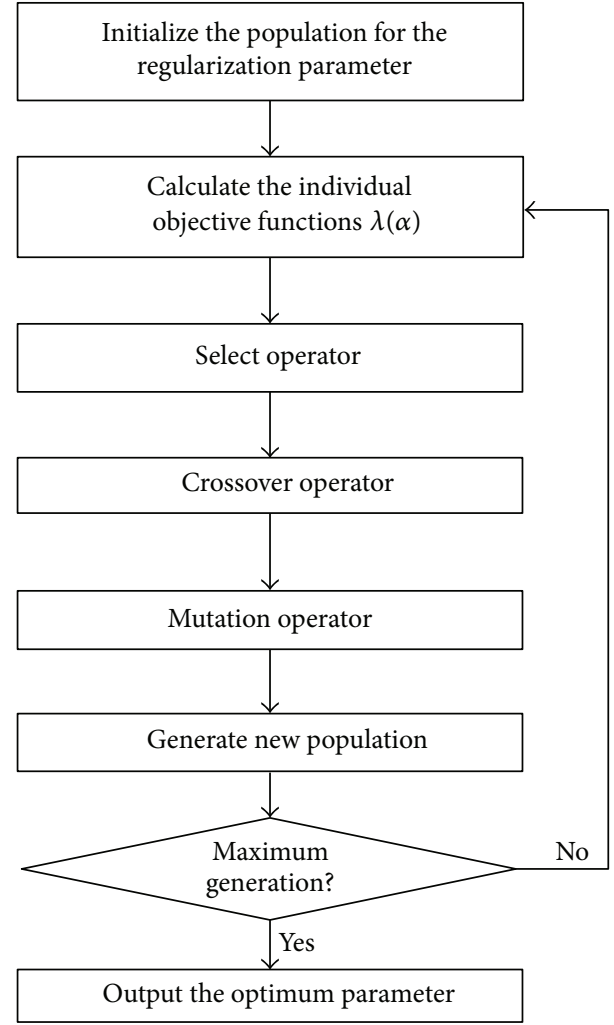

FIGURE 1: Flow chart for the calculation of regularization parameter based on GA.

of being selected for crossover. After selecting a pair of individuals, crossover is done by switching between the parts of the binary strings of these individuals. The population size remains constant through all the generations. This exchangedigit binary string corresponds in nature to the exchange of genetic information between parents, which represents the process by which it promotes the improvement of the species. The crossover for each pair of selected individuals is also subject to a probability $p_{c}$ to be performed. Mutation is a safeguard against losses of useful genetic material during reproduction and crossover. The process of mutation is simply to choose a few members from the population based on a probability of mutation $p_{m}$ and switch a 0 to a 1 and vice versa at a randomly selected site on the selected member. The process consisting of selection, crossover, and mutation is then repeated by the total number of generations, and at the end the most fitted individual is selected, that is, the one that leads to lower value of the objective function (31). The specific implementation process is shown in Figure 1.

\section{Numerical Examples}

In this section, we are going to demonstrate some numerical results for determining $f(x)$ in the inverse problems (1)(3). We use the $L_{\infty}$ error norm and the relative error to
TABLE 1: $T=0.1$, the objective function $\lambda(\alpha)$, the $L_{\infty}$, and the relative errors for $f(x)$, with $\delta=0,0.01,0.05,0.1$.

\begin{tabular}{lcccc}
\hline$\delta$ & $\alpha$ & $\lambda(\alpha)$ & $L_{\infty}$ & $\mathrm{RE}$ \\
\hline 0 & $6.7341 \times 10^{-10}$ & $8.6679 \times 10^{-9}$ & $5.8643 \times 10^{-6}$ & $5.8822 \times 10^{-6}$ \\
0.01 & $1.9422 \times 10^{-3}$ & 0 & $1.6741 \times 10^{-3}$ & $1.5696 \times 10^{-3}$ \\
0.05 & $9.7098 \times 10^{-3}$ & 0 & $1.9019 \times 10^{-3}$ & $1.7996 \times 10^{-3}$ \\
0.1 & $1.9419 \times 10^{-2}$ & 0 & $1.9352 \times 10^{-3}$ & $1.8332 \times 10^{-3}$ \\
\hline
\end{tabular}

TABle 2: $T=0.5$, the objective function $\lambda(\alpha)$, the $L_{\infty}$, and the relative errors for $f(x)$, with $\delta=0,0.01,0.05,0.1$.

\begin{tabular}{lcccc}
\hline$\delta$ & $\alpha$ & $\lambda(\alpha)$ & $L_{\infty}$ & $\mathrm{RE}$ \\
\hline 0 & $3.1837 \times 10^{-13}$ & $1.7318 \times 10^{-7}$ & $7.7189 \times 10^{-8}$ & $4.9543 \times 10^{-8}$ \\
0.01 & $3.7471 \times 10^{-5}$ & 0 & $3.2502 \times 10^{-6}$ & $3.2502 \times 10^{-6}$ \\
0.05 & $1.8735 \times 10^{-4}$ & 0 & $3.2502 \times 10^{-6}$ & $3.2502 \times 10^{-6}$ \\
0.1 & $3.7471 \times 10^{-4}$ & 0 & $3.2502 \times 10^{-6}$ & $3.2502 \times 10^{-6}$ \\
\hline
\end{tabular}

TABLE 3: $T=1$, the objective function $\lambda(\alpha)$, the $L_{\infty}$, and the relative errors for $f(x)$, with $\delta=0,0.01,0.05,0.1$.

\begin{tabular}{lcccc}
\hline$\delta$ & $\alpha$ & $\lambda(\alpha)$ & $L_{\infty}$ & $\mathrm{RE}$ \\
\hline 0 & $8.9302 \times 10^{-18}$ & $3.7471 \times 10^{-6}$ & $8.1049 \times 10^{-6}$ & $8.0927 \times 10^{-6}$ \\
0.01 & $2.6955 \times 10^{-7}$ & 0 & $2.5360 \times 10^{-4}$ & $2.5360 \times 10^{-4}$ \\
0.05 & $1.3477 \times 10^{-6}$ & 0 & $2.5464 \times 10^{-4}$ & $2.5464 \times 10^{-4}$ \\
0.1 & $2.6955 \times 10^{-6}$ & 0 & $2.5477 \times 10^{-4}$ & $2.5477 \times 10^{-4}$ \\
\hline
\end{tabular}

TABle 4: $T=1.2$, the objective function $\lambda(\alpha)$, the $L_{\infty}$, and the relative errors for $f(x)$, with $\delta=0,0.01,0.05,0.1$.

\begin{tabular}{lcccc}
\hline$\delta$ & $\alpha$ & $\lambda(\alpha)$ & $L_{\infty}$ & $\mathrm{RE}$ \\
\hline 0 & $6.1296 \times 10^{-16}$ & $2.9210 \times 10^{-6}$ & $7.4720 \times 10^{-7}$ & $7.4718 \times 10^{-7}$ \\
0.01 & $3.7452 \times 10^{-8}$ & 0 & $4.8134 \times 10^{-4}$ & $4.8134 \times 10^{-4}$ \\
0.05 & $1.8726 \times 10^{-7}$ & 0 & $4.8590 \times 10^{-4}$ & $4.8590 \times 10^{-4}$ \\
0.1 & $3.7453 \times 10^{-7}$ & 0 & $4.8647 \times 10^{-4}$ & $4.8647 \times 10^{-4}$ \\
\hline
\end{tabular}

measure the difference between the numerical and analytical solutions. The $L_{\infty}$ error norm is defined by

$$
L_{\infty}=\max _{0 \leq j \leq N}\left|f\left(x_{j}\right)-\tilde{f}\left(x_{j}\right)\right|,
$$

and the relative error (RE) is defined by

$$
\mathrm{RE}=\sqrt{\frac{\sum_{j=0}^{N}\left|f\left(x_{j}\right)-\tilde{f}\left(x_{j}\right)\right|^{2}}{\sum_{j=0}^{N}\left|f\left(x_{j}\right)\right|^{2}}},
$$

where $x_{j}$ are test points and $N$ is the total number of uniformly distributed points on $[0,1] . f(x)$ is the exact solution and $\widetilde{f}(x)$ is the numerical solution.

In our computations, we take $N=40$. The noisy data $\left.\left\{w^{\delta}\left(x_{j}\right)\right\}\right|_{j=0} ^{N}$ were assumed to contain some random errors. Assume the observed data has the following noised form:

$$
W^{\delta}=W+\delta \times \operatorname{randn}(\operatorname{size}(W)),
$$


TABLE 5: $T=1$, the analytical and numerical results for the $f\left(x_{j}\right)$ in $x_{j}=0.1 j$, where $\delta=0$.

\begin{tabular}{lccc}
\hline$j$ & Exact $f\left(x_{j}\right)$ & Numerical $\widetilde{f}\left(x_{j}\right)$ & $\left|f\left(x_{j}\right)-\widetilde{f}\left(x_{j}\right)\right|$ \\
\hline 1 & 0.309016994374947 & 0.309019506872923 & $2.51249797578179 \times 10^{-6}$ \\
2 & 0.587785252292473 & 0.587790008583465 & $4.75629099216945 \times 10^{-6}$ \\
3 & 0.809016994374947 & 0.809023584278051 & $6.58990310320462 \times 10^{-6}$ \\
4 & 0.951056516295154 & 0.951064106758891 & $7.59046373755012 \times 10^{-6}$ \\
5 & 1 & 1.00000810910255 & $8.10910254811503 \times 10^{-6}$ \\
6 & 0.951056516295154 & 0.951064262563094 & $7.74626794020072 \times 10^{-6}$ \\
7 & 0.809016994374947 & 0.809023549839842 & $6.5554648942534 \times 10^{-6}$ \\
8 & 0.587785252292473 & 0.587789998909465 & $4.74661699212398 \times 10^{-6}$ \\
9 & 0.309016994374948 & 0.309019477607716 & $2.48323276808016 \times 10^{-6}$ \\
10 & $1.22464679914735 \times 10^{-16}$ & $6.12328388409843 \times 10^{-17}$ & $6.1231841073751 \times 10^{-17}$ \\
\hline
\end{tabular}

TABLE 6: $T=1$, the analytical and numerical results for the $f\left(x_{j}\right)$ in $x_{j}=0.1 j$, where $\delta=0.1$.

\begin{tabular}{lcrc}
\hline$j$ & Exact $f\left(x_{j}\right)$ & Numerical $\tilde{f}\left(x_{j}\right)$ & $\left|f\left(x_{j}\right)-\widetilde{f}\left(x_{j}\right)\right|$ \\
\hline 1 & 0.309016994374947 & 0.308938263749532 & $7.8730625415413 \times 10^{-5}$ \\
2 & 0.587785252292473 & 0.587635497743805 & $1.49754548667946 \times 10^{-4}$ \\
3 & 0.809016994374947 & 0.808810874921651 & $2.06119453296294 \times 10^{-4}$ \\
4 & 0.951056516295154 & 0.950814208345435 & $2.42307949718912 \times 10^{-4}$ \\
5 & 1 & 0.999745222344235 & $2.54777655765204 \times 10^{-4}$ \\
6 & 0.951056516295154 & 0.950814208345429 & $2.42307949724685 \times 10^{-4}$ \\
7 & 0.809016994374947 & 0.808810874921642 & $2.06119453305731 \times 10^{-4}$ \\
8 & 0.587785252292473 & 0.587635497743796 & $1.49754548676939 \times 10^{-4}$ \\
9 & 0.309016994374948 & 0.308938263749526 & $7.87306254214082 \times 10^{-5}$ \\
10 & $1.22464679914735 \times 10^{-16}$ & $6.12167393253357 \times 10^{-17}$ & $6.12479405893996 \times 10^{-17}$ \\
\hline
\end{tabular}

where $W=\left(w\left(x_{0}\right), w\left(x_{1}\right), \ldots, w\left(x_{40}\right)\right)^{T}$, " $\operatorname{randn}(\cdot)$ " is a normally distributed random variable with zero mean and unit standard deviation, and $\delta$ dictates the level of noise. "randn $(\operatorname{size}(W)$ " returns an array of random entries that has the same size as $W$.

Example 1. In this example let us consider the following inverse problem:

$$
\begin{gathered}
\frac{\partial u}{\partial t}=\frac{\partial^{2} u}{\partial x^{2}}+\varphi(x, t), \quad 0<x<1, t>0, \\
u(0, t)=0, \quad t>0 \\
u(1, t)=0, \quad t>0, \\
u(x, 0)=f(x), \quad 0 \leq x \leq 1 .
\end{gathered}
$$

The overspecified condition is as follows:

$$
u(x, T)=g(x)=e^{-T} \sin (\pi x) .
$$

The analytical solution of this example is $u(x, t)=e^{-t} \sin (\pi x)$ and $\varphi(x, t)=\left(\pi^{2}-1\right) e^{-t} \sin (\pi x), f(x)=\sin (\pi x)$.

The regularization parameter $\alpha$ is chosen using the genetic algorithm. In the genetic algorithm implemented, a tournament selection scheme by two individuals was used. A uniform crossover operator is applied with a probability of 0.95 and a uniform mutation operator where each individual has a probability 0.05 of being mutated. Population size is 20 , number of generations is 100 . The search domain is $[0,100]$ for $\alpha$. The objective function $\lambda(\alpha)$, the $L_{\infty}$ error norm, and relative error RE are presented in Tables 1, 2, 3, and 4 corresponding to $T=0.1,0.5,1$, and 1.2 , respectively. Especially when $T=1$, the corresponding errors between the analytical values and the estimated results by function $f(x)$ are listed in Table $5(\delta=0)$ and Table $6(\delta=0.1)$.

From this numerical example, it can be seen that the numerical results are quite satisfactory. As shown in Tables 16 , when the noise level of input data $\delta=0$ with $T=0.1,0.5,1$, and 1.2, the numerical solutions obtained using our method are of high degree of accuracy. Even with the noise level of input data up to $\delta=0.1$, the numerical solutions are still in good agreement with the exact solutions.

\section{Conclusion}

This paper deals with the effective algorithms for solving the backward heat problem and the following results are obtained.

(1) The present study successfully applies the numerical method involving Tikhonov's regularization method 
in conjunction with the first Fredholm integral equation to the inverse nonhomogeneous heat conduction problems.

(2) From the illustrated example, it can be seen that the proposed numerical method is efficient and accurate to estimate the initial condition $f(x)$.

\section{Conflict of Interests}

The authors declare that there is no conflict of interests regarding the publication of this paper.

\section{Acknowledgments}

The work of the authors is supported by the Special Funds of the National Natural Science Foundation of China (nos. 51190093 and 51179151). The authors would like to thank the referees for constructive suggestions and comments.

\section{References}

[1] A. Shidfar, G. R. Karamali, and J. Damirchi, "An inverse heat conduction problem with a nonlinear source term," Nonlinear Analysis, Theory, Methods and Applications, vol. 65, no. 3, pp. 615-621, 2006.

[2] A. Shidfar and R. Pourgholi, "Numerical approximation of solution of an inverse heat conduction problem based on Legendre polynomials," Applied Mathematics and Computation, vol. 175, no. 2, pp. 1366-1374, 2006.

[3] J. Shi and J. Wang, "Inverse problem of estimating space and time dependent hot surface heat flux in transient transpiration cooling process," International Journal of Thermal Sciences, vol. 48, no. 7, pp. 1398-1404, 2009.

[4] B. T. Johansson and D. Lesnic, "A procedure for determining a spacewise dependent heat source and the initial temperature," Applicable Analysis, vol. 87, pp. 265-276, 2008.

[5] M. Ebrahimian, R. Pourgholi, M. Emamjome, and P. Reihani, "A numerical solution of an inverse parabolic problem with unknown boundary conditions," Applied Mathematics and Computation, vol. 189, no. 1, pp. 228-234, 2007.

[6] R. Model and U. Hammerschmidt, "Numerical methods for the determination of thermal properties by means of transient measurements," in Proceedings of the 6th International Conference on Advanced Computational Methods in Heat Transfer, pp. 407416, WIT Press, June 2000.

[7] R. Lattes and J. L. Lions, Methode de Quasi-Reversibilite et Applications, Dunod, Paris, France, 1967.

[8] H. Gajewski and K. Zacharias, "Zur regularisierung einer Klasse nichtkorrekter probleme bei evolutionsgleichungen," Journal of Mathematical Analysis and Applications, vol. 38, no. 3, pp. 784789, 1972.

[9] G. Nakamura, S. Saitoh, and A. Syarif, "Representations of initial heat distributions by means of their heat distributions as functions of time," Inverse Problems, vol. 15, no. 5, pp. 1255-1261, 1999.

[10] N. Al-Khalidy, "On the solution of parabolic and hyperbolic inverse heat conduction problems," International Journal of Heat and Mass Transfer, vol. 41, no. 23, pp. 3731-3740, 1998.
[11] J. V. Beck, B. Blackwell, and C. R. S. Clair, Inverse Heat Conduction: Ill-Poed Problems, Wiley Interscience, New York, NY, USA, 1985.

[12] E. Hensel, Inverse Theory and Applications for Engineers, Prentice Hall, 1991.

[13] D. T. Dang and H. T. Nguyen, "Regularization and error estimates for nonhomogeneous backward heat problems," Electronic Journal of Differential Equations, vol. 2006, pp. 1-10, 2006.

[14] D. D. Trong, P. H. Quan, T. V. Khanh, and N. H. Tuan, "A nonlinear case of the 1-D Backward heat problem: regularizai ion and error estimate," Zeitschrift fur Analysis und ihre Anwendung, vol. 26, no. 2, pp. 231-245, 2007.

[15] A. Hasanov, P. Duchateau, and B. Pektaş, "An adjoint problem approach and coarse-fine mesh method for identification of the diffusion coefficient in a linear parabolic equation," Journal of Inverse and Ill-Posed Problems, vol. 14, no. 5, pp. 435-463, 2006.

[16] Y.-F. Wang and T.-Y. Xiao, "Fast realization algorithms for determining regularization parameters in linear inverse problems," Inverse Problems, vol. 17, no. 2, pp. 281-291, 2001.

[17] G. D. Li and Y. F. Wang, "A regularizing trust region algorithm for nonlinear ill-posed problems," Inverse Problems in Science and Engineering, vol. 14, no. 8, pp. 859-872, 2006.

[18] V. A. Morozov, "Choice of a parameter for the solution of functional equations by the regularization method," Soviet Mathematics: Doklady, vol. 8, pp. 1000-1003, 1967.

[19] V. A. Morozov, Methods for Solving Incorrectly Posed Problems, Springer, Berlin, Germany, 1984.

[20] D. E. Goldberg, Genetic Algorithms in Search, Optimization and Machine Learning, Addison-Wesley, Reading, Mass, USA, 1989.

[21] P. Hajela, "Genetic search. An approach to the nonconvex optimization problem," AIAA Journal, vol. 28, no. 7, pp. 12051210, 1990.

[22] J. S. Arora, O. A. Elwakeil, A. I. Chahande, and C. C. Hsieh, "Global optimization methods for engineering applications: a review," Structural Optimization, vol. 9, no. 3-4, pp. 137-159, 1995. 


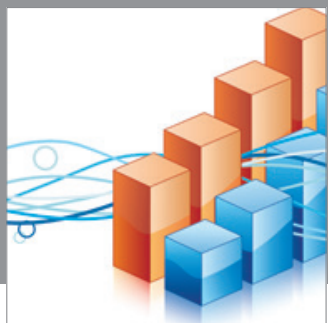

Advances in

Operations Research

mansans

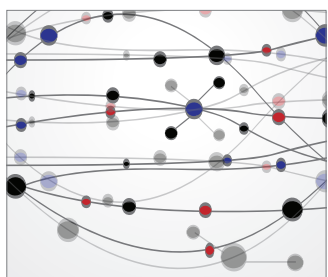

The Scientific World Journal
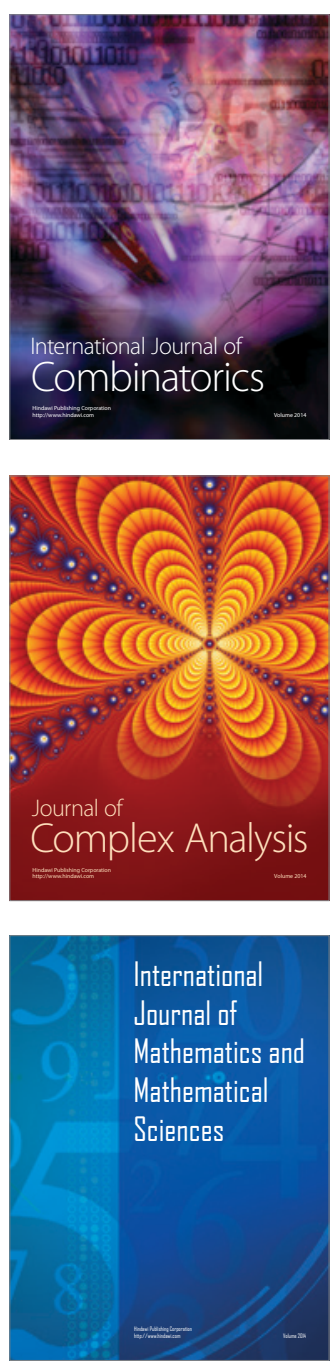
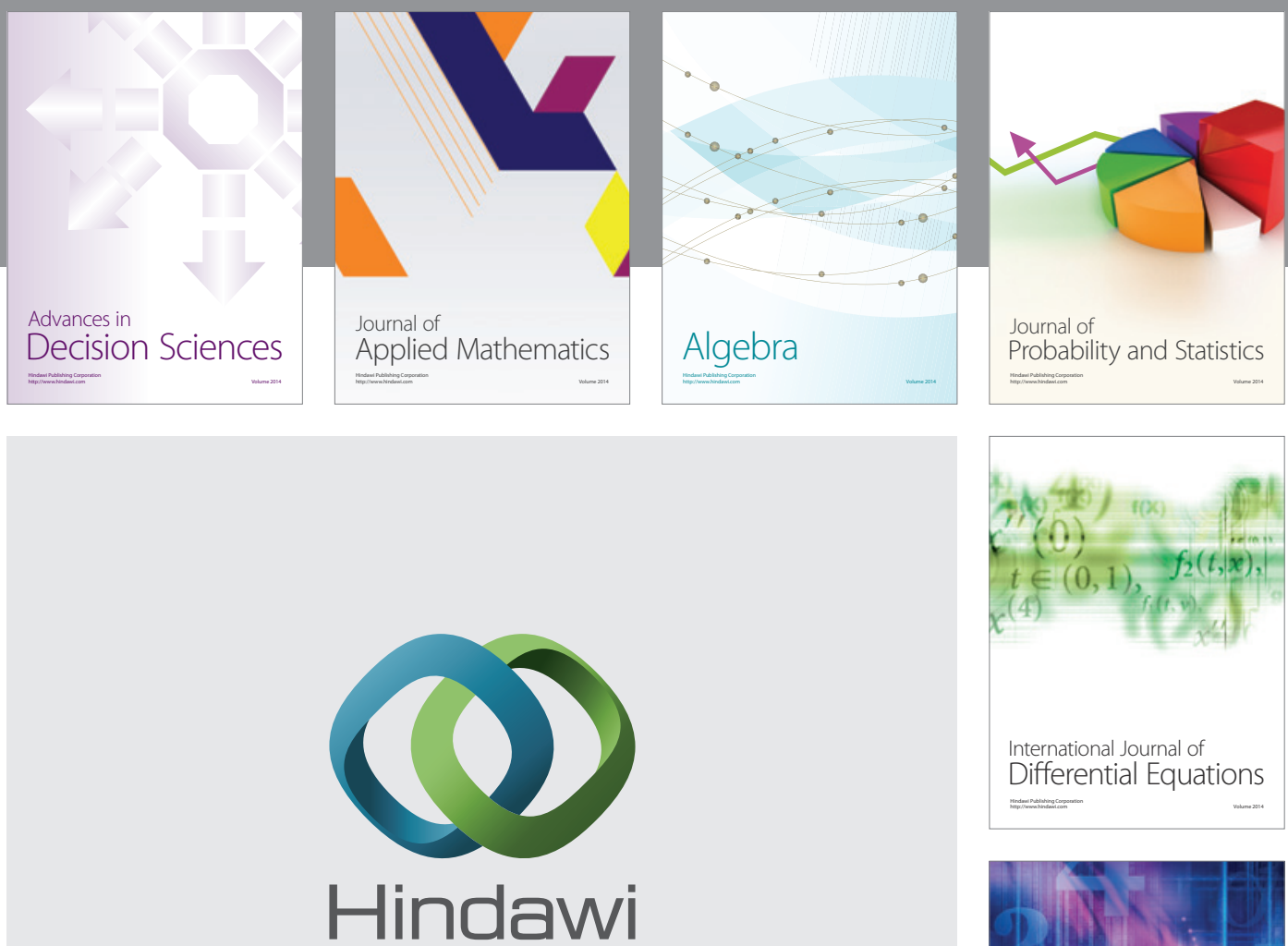

Submit your manuscripts at http://www.hindawi.com
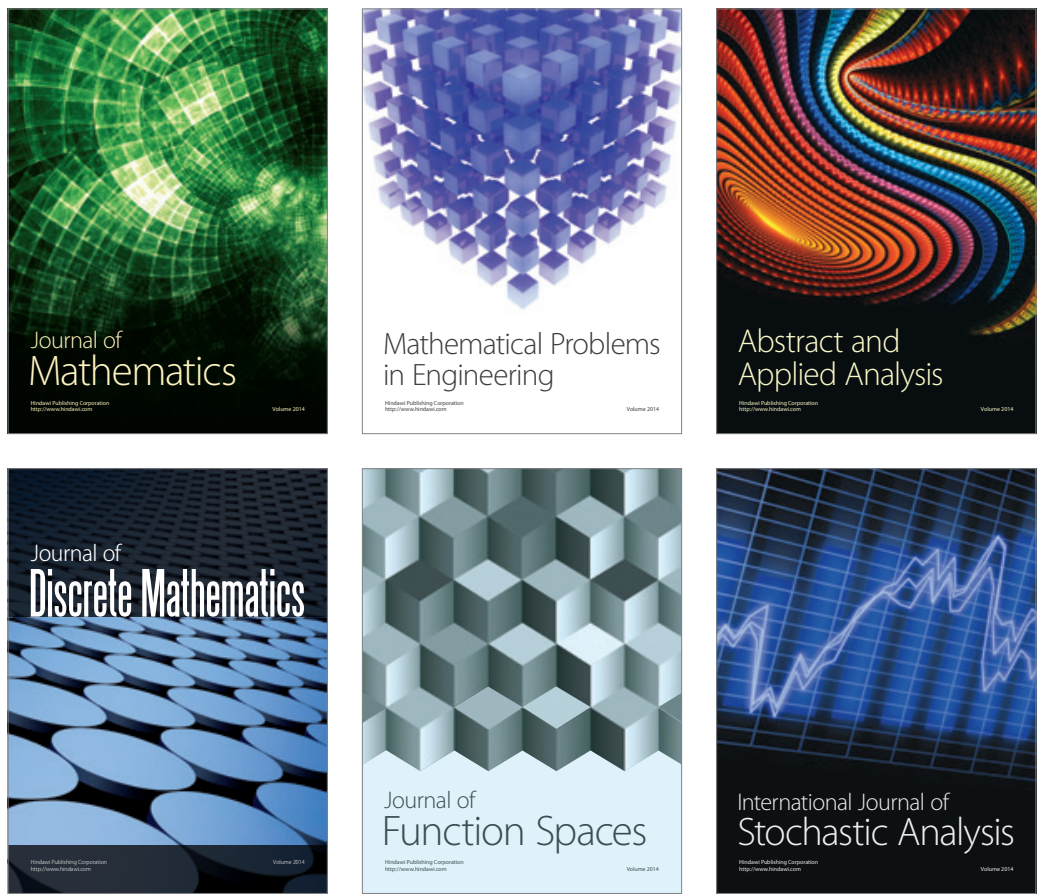

Journal of

Function Spaces

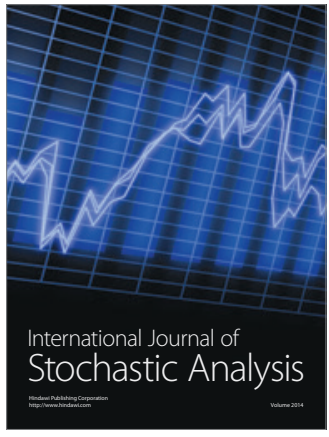

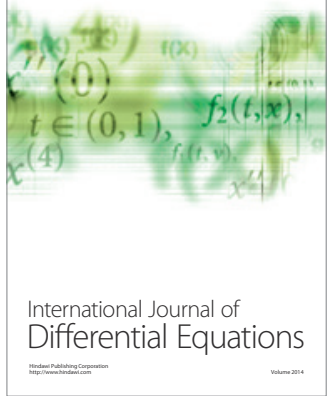
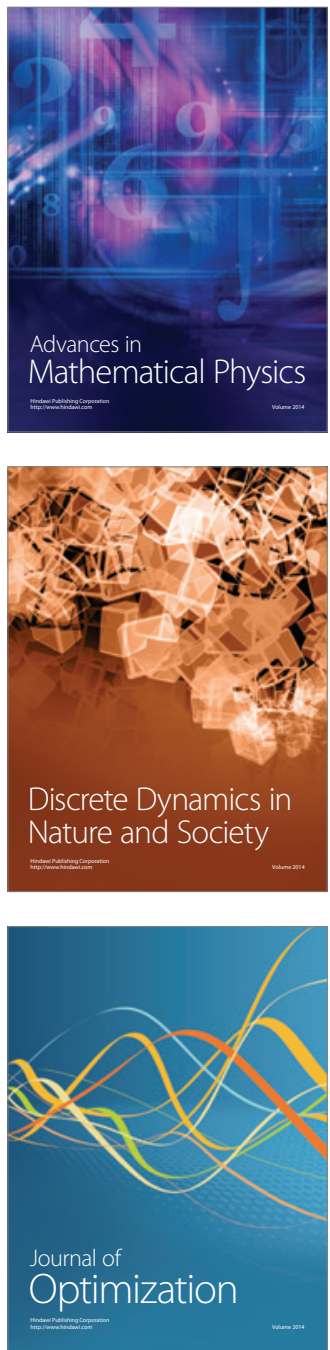